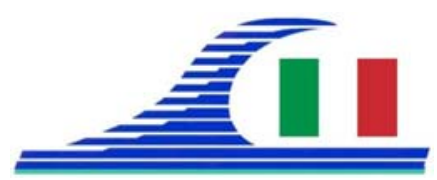

Conférence Méditerranéenne Côtière et Maritime

EDITION 3, FERRARA, ITALIA (2015)

Coastal and Maritime Mediterranean Conference

Disponible en ligne - http://www.paralia.fr - Available online

\title{
Technique non destructive innovante pour la détermination de la tension dans des tirants d'ancrage
}

\author{
Laurent MITAINE ${ }^{1}$, Jean-Jacques RINCENT ${ }^{2}$
}

1. Rincent Ports, 39 rue Michel-Ange 91026 Evry Cedex. rincentports@rincent.fr

2. Rincent BTP Services SAS, 39 rue Michel-Ange 91026 Evry Cedex. jean-jacques.rincent@rincent.com

\section{Résumé :}

Afin de faciliter la maintenance à long-terme des infrastructures maritimes et portuaires, cette technique de mesure non destructive de la tension s'applique aux tirants d'ancrage supportant aussi bien des quais en palplanches qu'en parois moulées.

Elle s’applique en réception de travaux ainsi qu’en suivi de tension résiduelle durant la vie de l'ouvrage.

Cette technique a trois bénéfices :

- Elle est non destructive, contrairement aux techniques habituelles par mise en tension complémentaire qui endommagent ou descellent $10 \%$ des tirants. Elle utilise une instrumentation par impédance mécanique, brevetée, mise en œuvre par un seul opérateur et sans aucun équipement lourd,

- Elle permet de tester rapidement une grande quantité de tirants, alors que les solutions classiques par chargement complémentaire, nécessitent plus de logistique et ne travaillent que par échantillonnage,

- Elle fonctionne aussi bien sous l'eau, par l'entremise d'un plongeur.

Cette technique permet donc de tester et de vérifier tous les tirants d'ancrage de quais (ou de tout autre type d'ouvrage utilisant des tirants), plus rapidement et dans des conditions de sécurité nettement plus satisfaisantes que les techniques habituelles par chargement.

Mots-clés : Génie côtier, Infrastructures Maritimes et Portuaires, Travaux maritimes, Quais, Tirants, Essais non destructifs, Fondations, Palplanches, Maintenance, Entretien.

\section{Introduction - Principe de fonctionnement de cette méthode non destructive}

La détermination non-destructive de la tension effectivement mise en œuvre après travaux - ou de la tension résiduelle dans le cas d'un diagnostic de maintenance - dans des tirants d'ancrage, est rendue possible par l'analyse de la réponse vibratoire du système mis en vibration au moyen d'un choc dont la force est mesurée.

Le système est ici assimilable à un ressort élastique qui a été mis en tension.

La réponse vibratoire obtenue est fonction : 
Côtes méditerranéennes menacées :

Risques et défis dans le contexte du changement climatique

- de la forme, de l'inertie, du diamètre du forage, de la longueur du tirant, de la nature des matériaux le constituant,

- des matériaux environnants (palplanche métallique, paroi moulée en béton armée, etc.),

- de l'état de contrainte du tirant.

\section{Matériels utilisés et modalités de mise en ouvre des essais}

Les moyens mis en œuvre (schématisés en figure 1) pour obtenir cette réponse vibratoire sont :

- un impacteur équipé d'un capteur de force, qui frappe dans l'axe du tirant,

- un capteur de vitesse ou d'accélération (de type géophone à aimant sur ressort) positionné sur la plaque d'appui de l'élément, ou à défaut sa tête d'ancrage,

- un traitement mathématique des acquisitions pour obtenir la courbe de fréquence (voir figure 2)

La procédure de l'essai consiste à :

1) accéder à la zone d'essai,

2) opérer la mise en station de l'opérateur qui pilote les acquisitions à partir d'un ordinateur de type PC portable,

3) brosser et nettoyer la plaque d'appui, préalablement à l'essai, pour permettre la fixation du capteur et l'impact du percuteur tous les deux sur une surface propre,

4) fixer le capteur par l'intermédiaire d'un produit couplant sur la plaque d'appui du tirant. Le produit couplant utilisé est de type graisse universelle au lithium, dont l'usage est validé par les autorités compétentes en termes d'hygiène et de santé au travail. Le capteur utilisé est adapté aux tirants inclinés (fonctionnement non symétrique),

5) mettre en vibration le tirant avec un impact provoqué sur sa plaque d'appui dans la direction de son axe. Pour chaque tirant testé, 6 acquisitions minimum sont réalisées, chacune étant le cumul de deux essais afin d'atténuer les vibrations parasites et d'amplifier la réponse vibratoire du tirant.

6) enregistrer la force d'impact $F$,

7) enregistrer la vibration V du système (tirant et palplanche ou paroi moulée) à l'aide du capteur fixé sur la plaque d’appui.

Mesures complémentaires à réaliser :

La détermination de l'effort de tension dans les tirants nécessite la réalisation d'essais non-destructifs complémentaires sur le bossage béton intermédiaire entre la palplanche / paroi moulée et la plaque d'appui en tête des tirants, afin d'identifier la part de raideur dynamique liée à la rigidité de ce bloc béton.

Le matériel et la procédure pour cet essai sont les mêmes que pour les essais sur les plaques d'appui. 
De même, des essais complémentaires sont effectués sur la palplanche ou la paroi moulée pour isoler leur part de raideur dynamique.

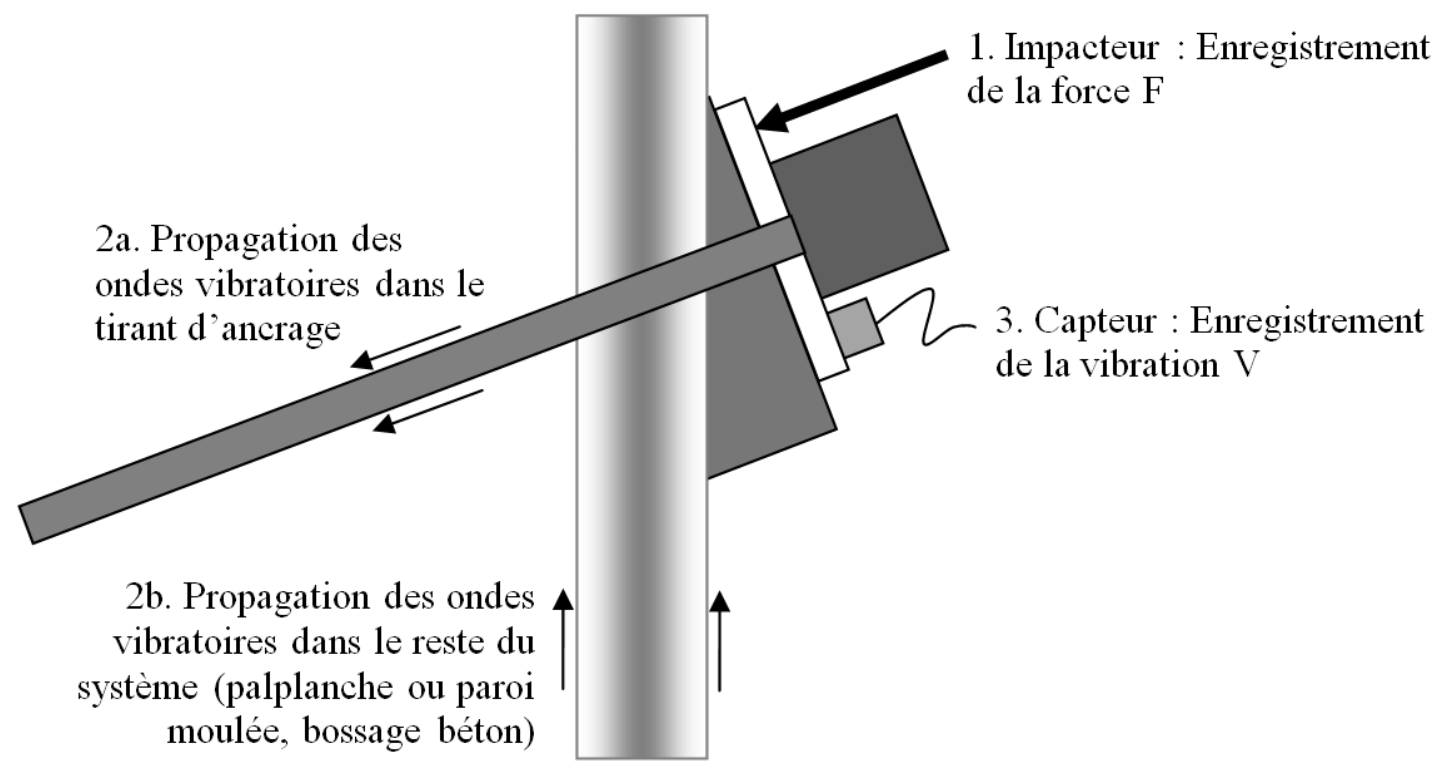

Figure 1. Principe de mise en œuvre des essais sur tirant d'ancrage.

\section{Détermination des longueurs par analyse de la réponse en fréquence}

L'analyse vibratoire porte sur les paramètres "réponse en fréquence" et "raideur " de la courbe de fréquence obtenue à la suite de l'acquisition (voir courbe type en figure 2).

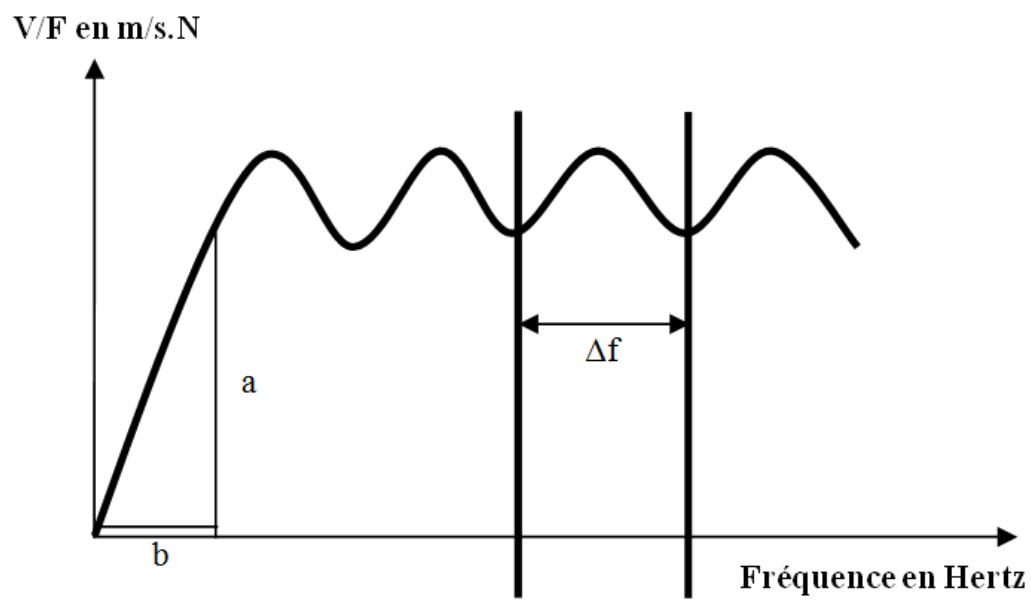

Figure 2. Courbe type après traitement des acquisitions.

Les différents pics ou creux successifs de la courbe sont positionnés en fréquence selon les fréquences propres des vibrations du système, selon le principe de la superposition de courbes sinusoïdales.

Décomposition des courbes : 
Côtes méditerranéennes menacées :

Risques et défis dans le contexte du changement climatique

Le théorème de Fourier définit que toute fonction périodique $f_{n}$ de période $\tau$ peut être écrite selon l'équation (1) :

$z(t)=\sum_{n} A_{n} \sin \left(2 \pi f_{n}+\phi_{n}\right)$

où $f_{n}=n f_{l}$ et $f_{l}=1 / \tau$

Dans ces conditions il est obtenu une courbe variant sous la forme d'une Vitesse/Force donnée en fonction de la fréquence.

La longueur de l'élément testé est obtenue par l'équation (2) :

$L=\frac{V}{2 \Delta F}$

où : $L$ est une longueur, $V$ la vitesse de propagation des ondes dans l'ancrage et $\Delta F$ l'écart de fréquence entre deux pics ou creux successifs.

Le ou les régimes vibratoires dominants conduisent à la détermination de la longueur totale du tirant et à sa longueur libre et de scellement le cas échéant, selon la figure 3.

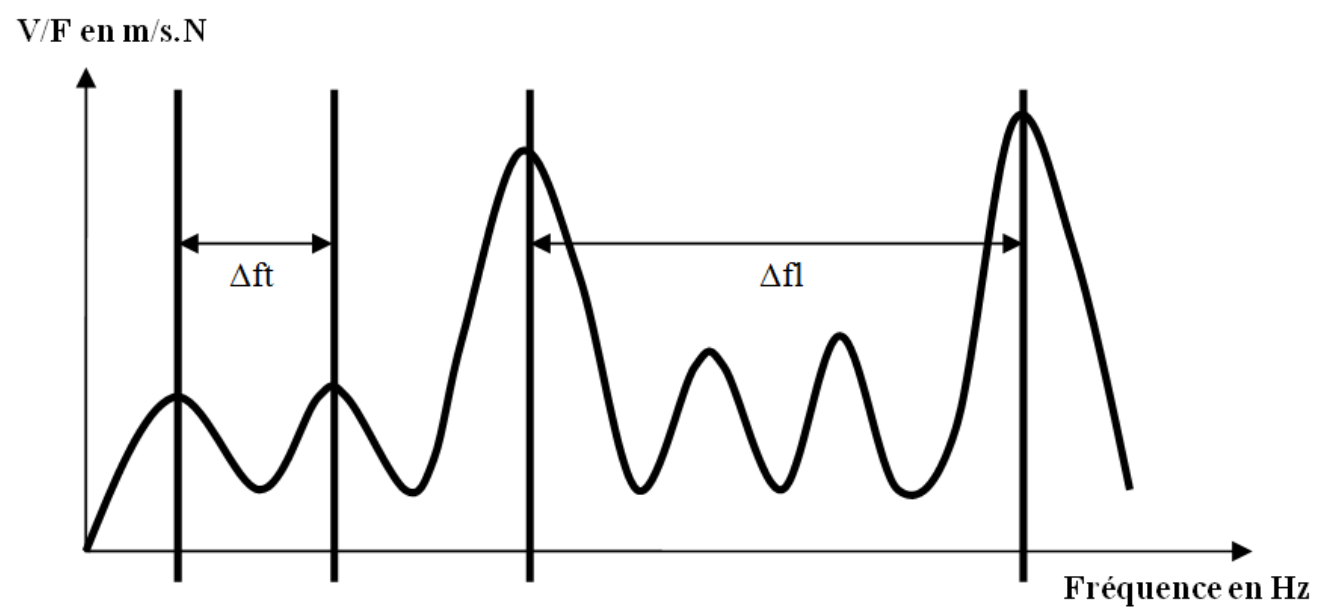

Figure 3. Principe de l'analyse des longueurs.

La mise en vibration de la partie libre de l'ancrage induit sur la courbe des pics ou creux successifs distant de $\Delta F_{l}$, soit une longueur $L_{l}$ (longueur libre) donnée par l’Eq. (3) :

$L_{l}=\frac{V}{2 \Delta F_{l}}$

De plus, la vibration de la totalité de l'ancrage donne sur la courbe des pics ou creux successifs distant de $\Delta F_{t}$, soit une longueur $L_{t}$ (longueur totale) donnée par l'Eq. (4) :

$L_{t}=\frac{V}{2 \Delta F_{t}}$

\section{Détermination de la raideur par analyse de la réponse en fréquence}

La raideur dynamique (exprimée en $\mathrm{N} / \mathrm{m}$ ), qui est proportionnelle à l'inverse de la pente de la courbe à son origine ( $2 \pi \mathrm{b} / \mathrm{a}$ en reprenant les valeurs de pente a et $\mathrm{b}$ depuis la figure 2), dépend de l’inertie du système ausculté et de son environnement. 
La raideur dynamique est un nombre complexe avec une partie réelle et une partie imaginaire, qui peut être représentée sous la forme d'un vecteur somme d'une raideur dans la direction orthogonale à l'application de la charge et d'une raideur dans la direction d'application de la charge.

La raideur statique est quant à elle la valeur de la pente en un point de la courbe effort/déformation, c'est à dire sous une charge donnée. Son unité est le $\mathrm{N} / \mathrm{m}$ et il s'agit de la valeur qui est recherchée.

La raideur dynamique d'un objet croît avec l'effort de tension qui est appliqué sur lui, et entraîne une réduction de l'amplitude de sa courbe de fréquence vibratoire, selon la figure 4 (les valeurs de tension indiquées pour chaque courbe sont données uniquement à titre d'exemple).

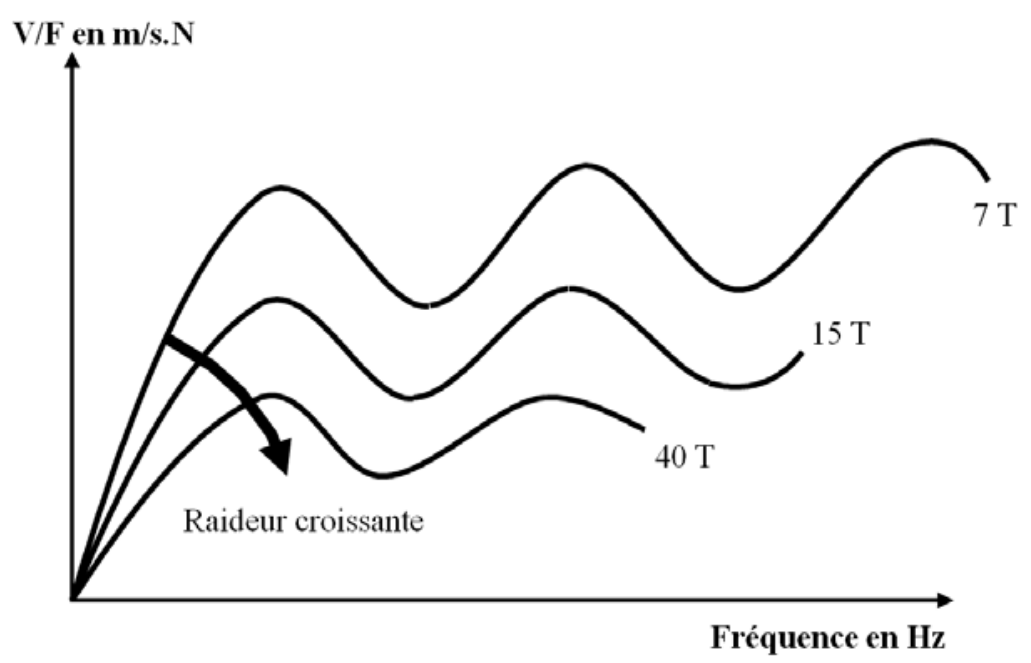

Figure 4. Réduction de l'amplitude des courbes de fréquence vibratoire d'un objet en lien avec l'augmentation de l'effort de tension appliqué sur lui.

Dans le cas d'un ancrage, elle dépend de son type et de ses caractéristiques géométriques, de son scellement et des efforts appliqués.

Une valeur de raideur "correcte" caractérise :

- un scellement correct de l'ancrage,

- l'absence de coupure,

- la continuité de l'ancrage sur sa longueur.

Une raideur faible met en évidence des désordres :

- au niveau de l'ancrage : une éventuelle coupure ou perte de tension,

- au niveau de son scellement.

L'expérience pratique acquise dans ce type de diagnostic montre que le ratio différentiel entre des mesures de raideurs "correctes" et de raideurs "faibles" est de l'ordre de 1 à 10 .

Ce paramètre est corrélable à la raideur statique, c'est-à-dire à la pente en un point de la courbe effort/déformation issue d'un essai de traction directe. 
Côtes méditerranéennes menacées :

Risques et défis dans le contexte du changement climatique

La raideur dynamique est donc liée à l'effort dans l'ancrage. La comparaison entre les raideurs obtenues sur les ancrages testés et les relations raideur dynamique / effort de tension ${ }^{\circledR}$ de la banque de données Rincent BTP Services permet d'estimer l'effort dans l'ancrage à l'issue de l'essai avec une marge d'erreur inférieure à 5\%.

\section{Conclusions}

A l'issue du dépouillement, les données de sortie obtenues sont :

- les longueurs libres et totales calculées et leur moyenne sur les acquisitions retenues,

- les raideurs mesurées et la moyenne sur les acquisitions retenues,

- l'interprétation de ces raideurs en effort de tension équivalent ${ }^{\circledR}$

Ce type d'essai et le brevet associé s’applique aux tirants, torons, barres, clous et plus généralement à tous types de renforcements scellés. Outre son utilité dans le domaine des infrastructures, il concerne aussi l'industrie avec par exemple la vérification des scellements de fixation de machines-outils.

De plus, il est à noter que ce type d'essai non destructif est adapté à la mesure in-situ de phénomènes de fatigue, puisqu'il permet de déterminer les variations de tension après l'apport de sollicitations cycliques, sans détruire l'élément testé.

Pour conclure, ce type d'essai non destructif permet de déterminer de façon précise et efficiente les caractéristiques les plus représentatives de tous types de tirant d'ancrage, en particulier dans le cadre des infrastructures maritimes et portuaires, et est utilisable comme technique instrumentée à la fois :

- pour la réception après travaux des tirants d'ancrage, donnant la possibilité d’un contrôle exhaustif et détaillé de tous les tirants réalisés,

- pour le suivi périodique dans le cadre de l'entretien et de la maintenance des tirants d'ancrage de structures de quai ou de soutènement existantes, avec une périodicité régulière et à moindre coût, qui permet de détecter les cas de sous-tension ou de surtension des tirants existants, et de prévenir les risques d'affaissement ou d'effondrement de parties d'ouvrages.

Sa rapidité d'exécution permet de mesurer de façon exhaustive un ensemble de tirants, là où les solutions classiques - qui nécessitent plus de logistique - ne travaillent que par échantillonnage.

\section{Bibliographie}

RINCENT BTP SERVICES (2006). Procédé et dispositif de détermination de l'effort de traction auquel est soumis un élément scellé. Brevet OMPI nWO 2006/010830 A1 déposé le 2 février 2006.

DAVIS A.G., DUNN C.S. (1975). From theory to field experience with the nondestructive vibration testing of piles. Ordinary meeting 25 February, 1975, at 5.30 p.m. Written discussion closes 7 March, 1975, for publication in Proceedings, Part 2. 